\title{
EchoGéo
}

\section{En attendant le centenaire... Introduction au dossier Afrique, 50 ans d'indépendance. Territoires, frontières, identités et développement}

\section{Géraud Magrin}

\section{CpenEdition}

Journals

Édition électronique

URL : https://journals.openedition.org/echogeo/12113

DOI : 10.4000/echogeo.12113

ISSN : 1963-1197

Éditeur

Pôle de recherche pour l'organisation et la diffusion de l'information géographique (CNRS UMR 8586)

Référence électronique

Géraud Magrin, «En attendant le centenaire... Introduction au dossier Afrique, 50 ans d'indépendance. Territoires, frontières, identités et développement », EchoGéo [En ligne], 13 | 2010, mis en ligne le 20 septembre 2010, consulté le 03 août 2021. URL : http://journals.openedition.org/echogeo/12113 ; DOI : https://doi.org/10.4000/echogeo.12113

Ce document a été généré automatiquement le 3 août 2021.

EchoGéo est mis à disposition selon les termes de la licence Creative Commons Attribution - Pas d'Utilisation Commerciale - Pas de Modification 4.0 International (CC BY-NC-ND) 


\title{
En attendant le
}

centenaire... Introduction au

dossier Afrique, 50 ans

d'indépendance. Territoires,

frontières, identités et

développement

\author{
Géraud Magrin
}

1 Ce dossier de la revue Echogéo se mêle à la saison de manifestations, notamment scientifiques, qui commémorent l'accession à l'indépendance de la plupart des États africains à la charnière des années 1960, il y a cinquante ans. Se conformant à la règle du genre, plusieurs colloques invitent à des réflexions en forme de bilan, qui ouvrent sur des fenêtres prospectives. Au sein d'une dominante historique, sociopolitique ou consacrée au développement ${ }^{1}$, les questions géographiques sont bien représentées. Elles procèdent tantôt de synthèses thématiques, tantôt d'orientations plus précises ou très ouvertes ${ }^{2}$.

2 Les dix textes ici présentés adoptent une focale assez large, qui couvre, dans une perspective le plus souvent diachronique, les relations entre territoires, frontières, identités et développement. Tous sont écrits en français; la plupart traitent de l'Afrique francophone - même si certains élargissent ponctuellement leur propos aux pays anglophones ou à l'ensemble du continent - ce qui témoigne du hiatus persistant entre les champs académiques francophones et anglophones, que l'appel à communication à l'origine du présent dossier essaya de transcender sans succès. Les auteurs ont par ailleurs des âges - certains ont commencé leur carrière peu après les indépendances, alors que d'autres sont presque nés à mi-étape, vers 1985 -, des parcours professionnels et des rattachements académiques variés, des centres d'intérêt qui le sont tout autant, ce qui explique la diversité de forme et de contenu des articles. 
Cette diversité se traduit surtout dans la nature des objets étudiés, et dans leur échelle : deux sont des villes. L'une, petite et méconnue (Bingerville, au sud de la Côte d'Ivoire), est observée dans le texte de Valéry Loba à travers les facteurs de son évolution du dernier demi-siècle, entre logiques institutionnelles et stratégies d'en bas, sur fond de conjonctures nationales fluctuantes et de réforme décentralisatrice. L'autre, Lomé, petite capitale au sein des grandes villes africaines, ayant intéressé de nombreux géographes (comme Émile Lebris, Philippe Gervais-Lambony, Benoit Antheaume, etc.), est abordée par Amandine Spire sous l'angle des pratiques de l'espace et des dynamiques identitaires associées à sa position frontalière. Les contributions de Najem Dhaher et Jean-Luc Piermay appréhendent les espaces nationaux tunisien et marocain, à travers notamment le rôle central de l'État dans les dynamiques de construction du territoire et d'ouverture à la mondialisation. Trois textes traitent d'un groupe de pays, autour de thèmes liés à des politiques publiques à forte implication spatiale: Éric Idelman et Jérôme Marie questionnent, en Afrique de l'Ouest, les changements dans l'exercice du pouvoir à l'échelle locale introduits par la décentralisation; Kirsteen Koop, Pierre-Antoine Landel et Bernard Pecqueur s'interrogent sur le sens du transfert des modèles français du développement territorial vers les pays du Maghreb; enfin, Camille Renaudin dresse un bilan prospectif des filières cotonnières des pays d'Afrique francophone - qui concernent une dizaine de pays d'Afrique de l'Ouest et du Centre. A partir d'une expérience d'observation participante, Armelle Choplin et Jérôme Lombard portent leur regard sur les circulations à travers les frontières de trois pays ouestafricains (Sénégal, Mali, Mauritanie), et sur ce qu'elles révèlent de l'interaction entre les pratiques trans-territoriales du bas et les logiques étatiques. Enfin, deux textes embrassent l'Afrique sub-saharienne dans sa totalité. Ils renvoient à sa relation avec des acteurs extérieurs, par le prisme de l'évolution comparée de l'aide bilatérale au développement française et britannique (Thierry Simon), et celui du phénomène d'achat de terres africaines par des investisseurs mondialisés (Florence Brondeau).

Ce dossier est organisé en deux ensembles. Un premier groupe de textes (Echogéo ${ }^{\circ} 13$ ) traite principalement de l'action de l'Etat sur le territoire (textes de J.L. Piermay ; N. Dhaher ; K. Koop, P.A. Landel et B. Pecqueur ; E. Idelman et J. Marie ; V. Loba). Le second (Echogéo $\mathrm{n}^{\circ} 14$ ), plus varié, aborde les thèmes des circulations et des identités, de l'agriculture, de l'aide au développement (articles d'A. Choplin et J. Lombard ; A. Spire ; C. Renaudin ; F. Brondeau ; T. Simon).

5 Pour mettre ces différentes contributions en perspective, nous commencerons ici par signaler l'importance du moment et de l'échelle de l'observation. Puis, nous montrerons comment ils illustrent, chacun à leur manière, la relation complexe entre l'État, les territoires et les politiques menées au nom du développement.

\section{Le moment et l'échelle}

6 Les commémorations appellent l'inventaire. Or, celui-ci est influencé par le contexte de sa réalisation. En 1985, le quart de siècle d'indépendances africaines (Carter, O'Meara, 1985) a des relents de gueule de bois de lendemain de fête (de l'indépendance) : sur fond d'une guerre froide qui délocalise quelques uns de ses conflits en Afrique, des gouvernements autoritaires justifient les difficultés des constructions nationales; la sécheresse sahélienne et ses famines accélèrent la fulgurante croissance urbaine, qui partout crée des mégapoles ingérables; l'échec des politiques de développement des 
années 1950-1960 se révèle avec le retournement des cours mondiaux des matières premières, déclenchant l'ajustement structurel. La réalité de l'indépendance est questionnée par la persistance des liens hérités, la géopolitique de l'aide, l'importation des modèles de développement, la dépendance économique persistante.

En 2010, le regard a changé. Après un temps de crises qui semblaient sans fin (Van de Walle, 2001), entre le cœur de l'ajustement structurel et des conflits d'une ampleur et d'une intensité encore inédite liés notamment aux pertes des rentes géopolitiques de la guerre froide (1989-2001), les années 2000 dessinent un contexte ambigu. L'intégration de l'Afrique dans la mondialisation qui s'accélère ${ }^{3}$ peut être lue comme une dimension de ces turbulences: les Plans d'ajustement structurel s'accompagnent d'un affaiblissement des États. La multiplication de projets aidés non coordonnés et les processus de décentralisation encouragés par les bailleurs de fonds aboutissent à la prolifération de territoires (collectivités locales, aires protégées et de projets) qui rendent le territoire (national) ingouvernable (Antheaume, Giraut, 2005). Parallèlement, la libéralisation économique encourage des investissements étrangers qui, sous formes d'enclaves (minières ou pétrolières, voire agro-industrielles ou forestières) mondialisées, parfois gouvernées par la violence, participent de ce vaste processus de fragmentation territorial, laissant les autres espaces en déshérence, aux conflits, aux organisations humanitaires ou sous la férule de pouvoirs néo-coutumiers (Ferguson, 2006).

8 Pourtant, un parfum d'années 1960 revient sur l'Afrique en cette fin de décennie 2000 (Magrin, 2010). L'annulation des dettes, les hauts cours des matières premières, l'afflux d'investissement étranger, la construction d'infrastructures, la consommation soutenue par les transferts des migrants, des relations assouplies avec les bailleurs de fonds - dans un contexte post-consensus de Washington en partie lié à l'arrivée de nouveaux partenaires, comme les pays émergents -, le regain d'État, définissent une conjoncture relativement favorable. Celle-ci conduit à s'interroger sur la possibilité de changements plus structurels. Jusqu'ici, l'espace et le vide - globalement, de très faibles densités démographiques -, expliquaient, sur le temps long, l'extraversion des pouvoirs africains, qui se contentaient de capter les flux avec l'extérieur plutôt que de ponctionner une production locale qu'ils renonçaient à encourager (Iliffe, 2009). En somme, la géographie s'opposait à la consolidation d'États territoriaux wébériens considérés comme indispensables au développement. La densification démographique et urbaine, ainsi que celle des infrastructures (Lombard, Ninot, 2010) - les routes nouvelles et la téléphonie mobile fournissent des technologies d'exercice du pouvoir à distance (voir Herbst, 2000) sans comparaison avec le niveau de 1960 -, changent la donne. De même, les transitions politiques internes (démocratisation) et les réformes dans la régulation de l'exploitation de ressources naturelles les plus fréquemment indexées dans les conflits (progrès de la transparence et des règles environnementales concernant l'exploitation des matières premières extractives, du bois (voir Rosser, 2006)), malgré leurs limites, semblent ouvrir des espaces de bifurcation (Capoccia, Kelemen, 2007) hors des trajectoires suivies jusqu'alors, qui s'étaient montrées chaotiques, extraverties, rentières et non porteuses de développement (Magrin, 2010).

Une autre habituelle difficulté de ce type d'inventaire tient à l'échelle d'observation. Peut-on prétendre parler de l'Afrique dans sa diversité à partir de dix textes portant sur des thèmes variés, à des échelles qui le sont tout autant? Évidemment non, même si, entre l'irréductibilité de l'étude de cas micro-locale et la réflexion stratosphérique 
continentale, il y a une place pour des analyses de niveau intermédiaire de grande utilité, dont procèdent la plupart des articles du dossier.

10 L'exercice du bilan pose aussi la question contiguë de la réalité de son objet : l'Afrique existe-t-elle? Pour la quarantaine d'États concernés, ces cinquante ans d'indépendance renforcent-ils l'existence d'une entité africaine «solidaire » - à travers une histoire commune marquée par les relations de dépendance et de domination subie vis-à-vis des pôles dominants du système monde, et l'expérience partagée du sous-développement -, ou bien au contraire cette durée a-t-elle été suffisante pour autoriser l'affirmation de spécificités nationales susceptibles de se traduire en trajectoires singulières divergentes? Les deux ne s'excluent pas, sans doute, mais il faudra attendre la prochaine commémoration pour en avoir confirmation.

11 Quoi qu'il en soit, entre le continent africain, la quarantaine d'États et les innombrables identités infranationales qui s'y inscrivent - ou en transcendent les limites à travers circulations et pratiques migratoires -, le niveau sous-régional offre un bon champ d'analyse. En Afrique du Nord, la Tunisie et le Maroc doivent-ils leur dynamisme économique et leur niveau de développement supérieur à ceux des autres pays d'Afrique à la proximité géographique (et donc migratoire et commerciale) avec l'Europe, ou à la force de leurs États? Ou bien à ces deux raisons, auxquelles s'ajouteraient d'autres facteurs? Une chose est sûre, la géographie des ensembles régionaux influence les systèmes de relation des États - au plan interne comme externe -, et constitue ainsi un paramètre important de leur évolution. De ce point de vue régional, les textes du dossier nous renseignent principalement sur des dynamiques maghrébines et ouest-africaines, moins précisément sur le reste du continent.

\section{État, territoires et développement}

12 Les politiques des États sur leur territoire - qui se donnent pour telles, ou à travers des politiques sectorielles à forte implication territoriale - reflètent la nature, la force ou la faiblesse relative des entités en question.

$13 \mathrm{Au}$ cours des cinquante ans écoulés, l'aménagement du territoire a connu très schématiquement deux moments: au temps de l'État fort des années 1960-1970, il s'agissait officiellement de résorber par des politiques volontaristes les déséquilibres issus de la décolonisation (extraversion des économies, des réseaux urbains et de transport) pour construire l'État-nation; à la faveur de la crise des années 1980, les enjeux ont glissé, avec les politiques libérales et les décentralisations, vers la promotion de l'insertion dans la mondialisation de territoires infranationaux dont on encourage l'expression des dynamismes propres, en association avec des politiques d'intégration régionale.

En Afrique du Nord, l'État a été la matrice de la dynamique des territoires. Au Maroc, comme le montre le texte de Jean-Luc Piermay, à partir d'une structure polycentrique héritée, le contrôle du centre sur les marges a constitué un objectif central de construction du territoire. L'ouverture économique sur le monde a paradoxalement renforcé l'autonomie nationale. En Tunisie, la vigueur des dynamiques économiques semble amplifier les déséquilibres antérieurs - la forte littoralisation de l'économie et les inégalités socio-spatiales, qui se traduisent localement par des tensions sur la terre et sur l'eau, comme l'expose Najem Dhaher. Mais la croissance économique d'ensemble concoure aussi, malgré la libéralisation, à la crédibilité de l'État. Dans les deux cas, la 
force du pouvoir central inhibe les autonomies locales, même si elles semblent plus favorisées au Maroc.

En Afrique de l'Ouest, l'ajustement structurel semble sonner le glas de l'action territoriale de l'État, en dehors de la poursuite de l'aménagement des grandes vallées sahélo-soudaniennes, dont le financement extérieur reste permis par les enjeux de sécurité alimentaire (Raison, Magrin, 2009). Mais la libéralisation ne produit pas qu'anarchie. Dans le secteur du transport, par exemple, l'explosion de la route - sur fond de crise du rail et de progrès des infrastructures, largement financées par l'aide amplifie les circulations d'une manière inédite (contribution d'Armelle Choplin et Jérôme Lombard). Cette dynamique constitue un puissant facteur de structuration du réseau des bourgs et villes secondaires dont l'importance relativement ancienne dans le processus de développement (Bertrand, Dubresson, 1997) semble croissante (Alvergne, 2008).

Dans le domaine des politiques agricoles, les débats contemporains autour de l'avenir des filières cotonnières d'Afrique francophone (article de Camille Renaudin) et de l'achat des terres par des acteurs extérieurs à l'Afrique (texte de Florence Brondeau) rappellent ceux qui agitaient les milieux du développement dans les années 1960. A l'époque, la culture cotonnière avait ses partisans et ses détracteurs. Les premiers dénonçaient l'extraversion et la dépendance - technologique et vis-à-vis de prix instables, spéculatifs -, des profits captés à l'extérieur, la concurrence de la culture de rente sur les systèmes agricoles antérieurs, pour l'eau, la terre, la force de travail, et donc les menaces sur la sécurité alimentaire qui en résultaient. Les seconds mettaient en avant la croissance macro-économique, la hausse du PIB et des exportations, la contribution à la balance des paiements, la rente versée à l'État ; mais aussi, localement, la construction d'infrastructures, la diffusion d'un progrès technique nécessairement synonyme d'intensification. Les achats de terres convoquent aujourd'hui des controverses exactement analogues.

Or, ces deux contributions montrent que la culture cotonnière telle qu'elle s'est diffusée en Afrique francophone et les achats de terres esquissés aujourd'hui par les investisseurs extérieurs entretiennent des rapports au territoire très différents, qui en disent long quant au changement de contexte. Le système cotonnier d'Afrique francophone, après la coercition initiale, et malgré les fragilités (internes et externes) qui rendent son avenir incertain, a constitué un des rares succès du développement : pan-territorial et spatialement structurant, il a favorisé la diffusion d'innovations techniques, la diversification des systèmes agricoles, l'essor du vivrier marchand (Chaléard, 1998). L'achat des terres, à l'inverse, procède de la logique d'enclaves spatialement encapsulées qui est celle des activités extractives : phénomène récent et mal connu, il présente encore plus d'opacité que l'exploitation pétrolière, et moins de régulations (Cotula et al., 2009). Les anticorps du contrôle citoyen ou de la société civile n'ont encore guère eu de prise sur des dynamiques dont la diffusion d'effets territoriaux positifs semble encore très aléatoire. La prise de ces " greffes » supposerait que les États définissent des règles du jeu favorables et les fassent respecter. Mais cette logique n'est-elle pas contradictoire avec celles, spéculatives et spasmodiques, voire géopolitiques, qui fondent l'attractivité de l'Afrique pour les puissances mondiales qui participent à cette dynamique, qu'elles soient publiques (États émergents ou pétroliers) ou privées (entreprises et fonds divers)? 
18 La crise des États des années 1980 s'accompagne de la promotion du local. Nouveau « fétichisme » du monde du développement (Dubresson, Jaglin, 2010), décentralisation et développement local se déclinent de conserve, dans des situations nationales auxquelles ils semblent plus ou moins adaptés. Cette vogue marque le retour d'un intérêt pour le développement à la base déjà manifesté à la charnière des années 1950-1960, autour du catholicisme social du père Lebret et de l'IRFED ${ }^{4}$ - qui avait notamment influencé le premier Premier ministre du Sénégal indépendant, Mamadou Dia (voir Colin, 2007) -, mais avait été effacé ensuite par la force du modèle de l'État jacobin. Les décentralisations semblent plus dynamiques en Afrique de l'Ouest - texte d'Éric Idelman et Jérôme Marie - qu'en Afrique du Nord. Parce que l'État central y est plus faible? Le lien n'a rien d'automatique : au Maroc, Jean-Luc Piermay montre que c'est près du centre que les concertations entre acteurs fonctionnent le mieux. Il y a là peut-être un enseignement à portée plus générale : on peut difficilement décentraliser ce qui n'a jamais été centralisé, il faut peut-être construire l'État avant de le décentraliser...

19 Décentralisation et développement local entretiennent des relations ambiguës (Dubresson, Fauré, 2005). En Afrique du Nord, le texte de Kirsteen Koop, Pierre Antoine Landel et Bernard Pecqueur montre que la faiblesse de la société civile a freiné la diffusion du modèle du développement local élaboré en France, dans les années 1980-1990. Au cours des années 2000, des projets de développement territorial - forme modernisée du développement local, qui prend en compte l'insertion dans des flux globaux, la complexité des modes de gouvernance, la valorisation des ressources spécifiques des territoires - sont en cours d'expérimentation. Ils correspondent à l'idée que la réflexion sur le développement renvoie désormais aux mêmes défis au Nord et au Sud: armer les territoires face à la mondialisation. En Afrique de l'Ouest, le développement local est omniprésent dans les discours et les intitulés des projets aidés depuis la fin des années 1990. Mais les dynamiques productives sont limitées et les articulations avec les acteurs de la décentralisation et de l'aide restent problématiques (Piveteau, 2005). L'importation profondément paradoxale - puisqu'il s'agit en théorie de stimuler des potentiels endogènes - d'un nouveau modèle de développement semble réitérer les travers anciens de l'aide et s'inscrire dans une histoire africaine de l'extraversion (Bayart, 2006) où tout change pour que rien ne change.

L'aide au développement demeure d'ailleurs un sujet de débat vivace - comme le montre par exemple le récent pamphlet de Dambisa Moyo (2009) et la réponse de JeanMichel Severino et Jean-Michel Debrat (Severino, Debrat, 2009). Décriée pour ses motifs géopolitiques et les liens clientélistes hérités de l'ère coloniale qu'elle véhicule, l'aide bilatérale eut tout de même le mérite de soutenir les États quand l'aide multilatérale, avec ses approches projets et ses agences parallèles, tendait à les déconstruire, comme le montre le texte de Thierry Simon. Celui-ci retrace l'évolution géographique de l'aide bilatérale française et britannique depuis 1960. Alors que les pratiques de ces deux puissances moyennes suivent des tendances apparemment inverses - le champ français s'élargit, sur fond de tâtonnement institutionnel et de réduction des volumes financiers, tandis que l'aide britannique tend à se concentrer sur un petit nombre de pays parmi les plus pauvres, situés notamment en Afrique -, l'irruption de nouveaux acteurs (les pays émergents, et notamment la Chine) définit un nouveau contexte. Celui-ci est porteur de risques (en termes de gouvernance, d'atomisation, de 
contradictions) mais aussi d'opportunités (augmentation des volumes de financement, mise en concurrence et gains d'autonomie pour les États africains).

Enfin, les relations entre États modernes, encadrements préexistants et stratégies sociales endogènes sont centrales pour rendre compte des dynamiques territoriales africaines des dernières décennies, en lien avec celles des identités. En effet, l'existence même du territoire de l'État stimule des dynamiques du bas qui se jouent de lui. Les frontières éloignent ce qui est proche et rapprochent ce qui est éloigné; elles constituent des axes de structuration importants (Igué, 2006). La contribution d'Amandine Spire montre ainsi comment, à Lomé, la frontière où campe la ville ouvre un champ fécond de métissages spatiaux et d'hybridation identitaires. Dans ce texte comme dans celui d'Armelle Choplin et Jérôme Lombard, les savoir-circuler à travers les frontières illustrent les subtilités de la fabrique identitaire africaine, ainsi que le potentiel économique associé à ces espaces de l'entre-deux qui disent à la fois la présence de l'État et ses dysfonctionnements.

En outre, les relations entre pouvoir de l'État et pouvoirs antérieurs se déclinent suivant une grande diversité de formes (Perrot, Fauvelle-Aymard, 2003). Le Maroc étudié par J.L. Piermay est original : le pouvoir néo-traditionnel du Mahzen se confond après l'indépendance avec l'État moderne, ce qui n'est pas sans en faire la force. Rien de tel en Afrique de l'Ouest. En revanche, la contribution d'Éric Idelman et Jérôme Marie montre qu'un des enjeux majeurs des processus de décentralisation y consiste justement à prendre en charge le schisme institué à l'époque coloniale et perpétué à l'indépendance entre le droit foncier moderne et les pratiques paysannes. Les collectivités locales se trouvent ainsi priées de faire la synthèse entre le pouvoir de l'État et les encadrements préexistants, ce qui fonctionne plus ou moins bien. A Bingerville, étudiée par Valéry Loba, un tissu urbain composite illustre les tentatives planificatrices de l'État des années 1960-1970 contrées par la résistance des autochtones Ébrié, qui lotissent informellement en retour. La décentralisation rend finalement possible une reprise de la fabrique urbaine, sur fond de coalescence des fronts d'urbanisation entre les banlieues de la mégapole abidjanaise et ce qui fut longtemps une petite ville somnolente.

\section{Attendre encore 50 ans pour le développement ?}

" Il faut du temps pour que le sucre fonde, il faut du temps pour le développement ", disait Roland Pourtier en pastichant Bergson. Les macro-économistes peuvent toujours attendre (encore cinquante ans?) que l'heure de l'Afrique advienne, dans un jeu mondial où la hausse des salaires de l'Asie développée (après l'Amérique latine) viendrait enfin sonner le temps de l'essor du continent noir - les masses à présent conséquentes de citadins pauvres devenant attractives pour le capitalisme industriel mondial, si tant est que ses formes ne soient pas devenues d'ici là plus économes en travail humain, comme cela semble vraisemblable.

En attendant, les incertitudes actuelles rendent toujours aussi passionnante l'étude de l'Afrique mondialisée. Scruter les hybridations des logiques du dedans et du dehors, les interactions des stratégies de l'État et des acteurs d'en bas, les jeux subtils des identités et des territoires, de la dépendance et de l'autonomie, conduira à interroger, peut-être, les signes d'individualisation de trajectoires nationales plus favorables. On cherchera à y déchiffrer, quoi qu'il en soit, les pages d'une histoire indépendante complexe qui se 
sédimente dans des espaces aux changements d'une ampleur et d'une rapidité inédites. C'est à cet exercice que vous convient ces dix textes de géographes, en attendant ceux qui se pencheront, d'ici cinquante ans, sur un prochain bilan.

\section{BIBLIOGRAPHIE}

Alvergne C., 2008. Le défi des territoires. Comment dépasser les disparités spatiales en Afrique de l'Ouest et du Centre? Karthala, PDM, $264 \mathrm{p}$.

Antheaume B., Giraut F. (dir.), 2005. Le territoire est mort, vive les territoires! Une (re)fabrication au nom du développement. Paris, IRD Éditions.

Bayart J.F., 2006. «Comme vous en Afrique », ou l'hégémonie dans l'extraversion , préface à la nouvelle édition de L'État en Afrique. La politique du ventre. Paris, Fayard 1989 : III-LXVIII.

Bertrand M., Dubresson A. (dir.), 1997. Petites et moyennes villes d'Afrique noire. Paris, Karthala, $326 \mathrm{p}$.

Capoccia G., Kelemen R.D., 2007. The Study of Critical Junctures: Theory, Narrative and Counterfactuals in Historical Institutionalism. World Politics, vol. 59, Number 3, April 2007, p. 341-369.

Carter G.M., O'Meara P. (eds.), 1985. African Independence. The First Twenty-Five Years. Bloomington, Indiana University Press, $364 \mathrm{p}$.

Chaléard J.L., 1998. Temps des villes, temps des vivres. L'essor du vivrier marchand en Côte d'Ivoire. Paris, Karthala, 664 p.

Colin R., 2007. Sénégal notre pirogue. Au soleil de la liberté. Journal de bord 1955-1980. Paris, Présence africaine, $405 \mathrm{p}$.

Cotula L., Vermeulen S., Leonard R., Keeley J., 2009. Land Grab or Development Opportunity? Agricultural Investment and International Land Deals in Africa. IIED/FAO/IFAD, London, Rome.

Dollfuss O., 1997. La mondialisation. Paris, Presses de Sciences Po., 166 p.

Dubresson A., Fauré Y.A. (dir.), 2005. Décentralisation et développement local, un lien à repenser. Tiers Monde $\mathrm{n}^{\circ}$ 181, tome XLXI, janvier-mars 2005, $239 \mathrm{p}$.

Dubresson A., Jaglin S., 2010. Villes et citadins d'Afrique noire francophone. Le temps des incertitudes. Pourtier R. (dir.), 1960-2010. Un demi-siècle de mutations africaines, BAGF, 2010-1, p. 15-25.

Ferguson J., 2006. Global shadows. Africa in the neoliberal World Order. Durham and London, Duke University Press, 257 p.

Herbst J., 2000. States and Power in Africa. Comparative Lessons in Authority and Control. Princeton University Press, 280 p.

IguéJ.O., 2006. L'Afrique de l'Ouest entre espace, pouvoir et société. Une géographie de l'incertitude. Paris, Karthala, 555 p. 
Iliffe J., 2009. Les Africains. Histoire d'un continent. Flammarion, Champs histoire, 701 p. (titre original: Africans: The History of a Continent, Cambridge University Press, 1995 et 2007).

Lombard J., Ninot O., 2010. Connecter et intégrer : les territoires et les mutations des transports en Afrique. Pourtier R. (dir.), 1960-2010. Un demi-siècle de mutations africaines, BAGF, 2010-1, p. 69-86.

Magrin G., 2010. Rentes, territoires et développement. Que tout change pour que rien ne change? Pourtier R. (dir.), 1960-2010. Un demi-siècle de mutations africaines, BAGF, 2010-1, p. 56-68.

Moyo D., 2009. L'aide fatale : les ravages d'une aide inutile et de nouvelles solutions pour l'Afrique. Paris, J.C. Lattès, 280 p.

Perrot Cl.H., Fauvelle-Aymard F.-X. (dir.), 2003. Le retour des rois, les autorités traditionnelles et l'État en Afrique contemporaine. Paris, Karthala, 568 p.

Piveteau A., 2005. Décentralisation et développement local au Sénégal : chronique d'un couple hypothétique. Tiers monde, vol. XLVI, n 181, p. 71-93.

Pourtier R. (dir.), 2010. 1960-2010 : 50 ans d'indépendances africaines, dossier du BAGF, mars 2010, 160 p.

Raison J.P., Magrin G. (dir.), 2009. Des fleuves entre conflits et compromis. Essais d'hydropolitique africaine. Paris, Karthala, 299 p.

Rosser A., 2006. The Political Economy of the Resource Curse: A Literature Survey. IDS Working paper $\mathrm{n}^{\circ} 268$, Centre for the Future State, $34 \mathrm{p}$.

Severino J.M., Debrat J.M., 2009. L'aide au développement. Paris, Le Cavalier Bleu, 127 p.

Van de Walle N., 2001. African Economies and the Politics of Permanent Crisis 1979-1999.

Cambridge University Press.

\section{NOTES}

1. Par exemple les colloques «Afrique. 50 ans d'indépendance. Bilan et perspectives », Université Paris Ouest Nanterre, 3-4 juin 2010 ; «Recherches et débats : réinventer l'Afrique ? $2^{\text {ème }}$ congrès du Réseau des Etudes africaines en France, Bordeaux, 6-7-8 septembre 2010, CEAN, IEP Bordeaux ; ou encore « Afrique. 50 ans d'indépendance. Développement et coopération : quelles perspectives d'avenir? ", Revue internationale de politiques de développement et Afrique contemporaine, 29 octobre 2010.

2. Cf. le colloque "1960-2010: 50 ans d'indépendances africaines", Paris, 9 janvier, 2010, Association des géographes français (voir Pourtier, 2010) ; et « Frontières et indépendances en Afrique », Paris, INHA, 21-22 mai 2010.

3. L'Afrique participe de la mondialisation depuis son origine au XVe siècle (Dollfus, 1997). L'accélération caractéristique de la mondialisation contemporaine l'affecte comme le reste du monde, sous des formes spécifiques qui tiennent aux relations asymétriques de son insertion dans le système mondial. Elle coïncide avec la période dramatique 1989-2001.

4. Institut de recherche, de formation et de développement. 


\section{AUTEUR}

\section{GÉRAUD MAGRIN}

Géraud Magrin, geraud.magrin@cirad.fr, est chercheur Cirad (UMR Tetis) / UMR Prodig. Il a publié récemment :

- Magrin G., 2010. Rentes, territoires et développement. Que tout change pour que rien ne change ? Pourtier R. (dir.), 1960-2010. Un demi-siècle de mutations africaines, BAGF, 2010-1, p. 56-68.

- Magrin G., van Vliet G., 2009. The Use of Oil Revenues in Africa. In Lesourne J. (ed.), Governance of Oil in Africa: Unfinished Business, Paris. Ifri, Les Etudes, Gouvernance européenne et géopolitique de l'énergie, Tome 6, p. 103-163. www.ifri.org/files/Energie/MAGRIN.pdf - Raison J.P., Magrin G. (dir.), 2009. Des fleuves entre conflits et compromis. Essais d'hydropolitique africaine; Paris, Karthala, 299 p. 